RESEARCH PAPER RP842

\author{
Part of Journal of Research of the National Bureau of Standards, Volume 15, \\ November 1935
}

\title{
THE WAIDNER-WOLFF AND OTHER ADJUSTABLE ELECTRICAL-RESISTANCE ELEMENTS
}

\author{
By E. F. Mueller and Frank Wenner
}

\begin{abstract}
The Waidner-Wolff adjustable resistance element has to a considerable extent been used in resistance-measuring apparatus and potentiometers. It serves to change the resistance of a circuit in a number of small and equal steps by means of a dial switch, at the same time reducing the effect of variations of resistance of the switch contacts and thermoelectromotive forces in the switch. The Waidner-Wolff element, modifications of it, and another arrangement serving substantially the same purpose are considered in some detail. A three-terminal element used in bridge circuits and a four-terminal parallel element are also described. The adaptability of the parallel element to form useful combinations with similar or different elements is illustrated by examples. Finally, there is a list of the more important adjustable resistance elements now available to the designer of apparatus.
\end{abstract}

I. Introduction

II. The Waidner-Wolff element

III. Modifications of the Waidner-Wolff element..... 483

IV. The fixed-shunt element...... 486

V. The movable-branch-point element_._. 487

VI. The parallel element

VII. Combinations of elements_...... 488

VIII. Elements developed elsewhere

IX. List of adjustable resistance elements..... 492

X. Bibliography

\section{INTRODUCTION}

From time to time, since the organization of the National Bureau of Standards in 1901, occasions have arisen requiring the use of a combination of electrical resistors the resistance of which may be changed over a limited range in a large number of equal steps of desired magnitude. These changes might be made by the use of a suitable number of dial switches with contacts directly in the circuit, as in the ordinary dial resistance box. However, in many cases, variations of the resistances of the switch contacts are larger than is permissible in the resistor, or even larger than one of the steps. Also, in many cases, the thermoelectromotive forces in the switches are larger than is permissible in the resistor. Both of these difficulties have been obviated or materially reduced by placing the switch contacts in a shunt having a resistance several times that of the resistance of that part of the resistor shunted. Since each dial switch and the resistance 
sections directly associated with it may be considered more or less independently of the others, each will be referred to as an adjustable resistance element. While some of the arrangements used or proposed have been described elsewhere, others have not been described or the descriptions are not sufficiently complete to give a definite understanding of their advantages and limitations. It is the purpose of this paper to give a connected discussion of the different adjustable resistance elements developed in this Bureau as well as to provide a list of the more important ones now available for use in design of resistance apparatus.

\section{THE WAIDNER-WOLFF ELEMENT}

The Waidner-Wolff resistance element or decade was devised at the National Bureau of Standards about 1902 by C. W. Waidner and F. A. Wolff. It consists essentially of a relatively small fixed resistance shunted by another larger resistance, the latter variable by means of a dial switch, in 10 unequal steps, so chosen that the resultant resistance is variable in 10 equal steps, and for the highest step the resistance of the shunt is infinite.

One of the advantages of the element is that the values of the resultant equal resistance steps depend upon the values of comparatively large resistances which need be adjusted to an accuracy only slightly higher proportionally than that desired for the resistance steps. As the switch is in a shunt circuit of relatively high resistance, variations of the resistance of switch contacts, or electromotive forces originating in the switch, produce only a small fraction of the effect that they would produce if the switch were directly in the main circuit. Thus it is possible to have a decade with definite steps, even when the ordinary variations in the contact resistance of the switch are larger than the steps themselves. A disadvantage is that the resistance of the element cannot be reduced to zero.

In one of the original applications, three such elements in series, providing steps of $0.001,0.0001$, and $0.00001 \mathrm{ohm}$, respectively, were used as a substitute for the slide wire previously used in the Callendar-Griffiths bridge for resistance thermometry, as described by Griffiths (see bibliography for references). In another application, four elements, the smallest with steps of $0.000001 \mathrm{ohm}$, were used in one of the two ratio arms of a Wheatstone bridge or a Thomson double bridge. Apparatus embodying both of these applications was delivered to this Bureau in 1903.

The element has since been used to a considerable extent in resistance-measuring apparatus and potentiometers. It was first described very briefly by Waidner and Dickinson. It was described in detail by White as a well-known device of presumably remote origin, and has been mistakenly attributed to nim by several writers, although he explained its origin in a later publication.

The element is illustrated in figure 1 , in which the sketch marked " $A$ " represents the element with the customary marking when it is connected to provide 10 resistance increments of $0.01 \mathrm{ohm}$ each. The sketch marked " $\mathrm{B}$ " shows the notation to be used in the subsequent discussion. The reason why this notation is preferred for purposes of calculation will become self-evident in connection with the calculations for the modified Waidner-Wolff element. In some applications, the element would actually be marked as shown in 
sketch B. When two or more elements are used in series, the marking $\mathrm{X}$ is preferred to the marking 10 , because $\mathrm{X}$ may be recorded as if it were a digit, and this has several advantages.

The fixed resistance of the element is represented by $r$, which is a four-terminal conductor, so that the resistance included in it is definitely fixed by the positions of the branch points. When the switch is on the 0 position of figure $1 \mathrm{~B}$, the resistance of the element is $r$. For the switch positions 1, 2, etc., the corresponding resistance of the entire shunt, including connecting resistances and switch contacts, is designated as $S_{1}, S_{2}$, etc. If the connecting and contact resistances omitted are exactly equal to those introduced when the switch is moved, for example from 3 to 4 , then $S_{3}-S_{4}$ is equal to ${ }_{3} \mathrm{~A}_{4}$. According to the notation used here, ${ }_{3} \mathrm{~A}_{4}$ designates the drop in potential from 2 to 4 divided by the current in the shunt with the switch on position 3. It will be convenient to speak of the difference between the resistance of $r$ and the combination of $r$ shunted by $S$, as the "lowering", $L$. Thus $L_{0}, L_{1}, L_{2}$, etc., are the lowerings corresponding to switch positions $0,1,2$, etc. Since the shunt $S_{0}$ is infinite, $L_{0}=0$. Since all the steps are equal, each step is also equal

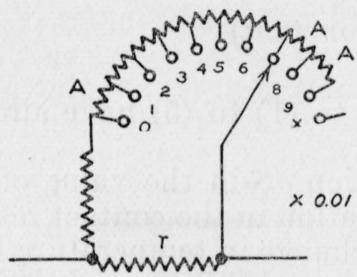

$A$

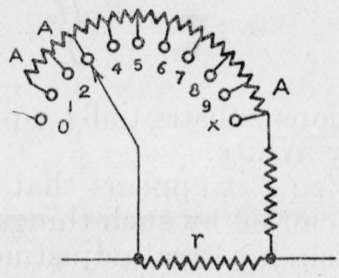

B

FIGURE 1.-Waidner-Wolff element

A. Element with customary marking for 10 resistance increments of $0.01 \mathrm{ohm}$ each.

B. Element marked to show notation used in discussion and calculations, illustrative of lowerings in ten equal steps.

to $L_{1}$. The resistance of a circuit which includes the element shown in figure 1 cannot be reduced below $r-10 L_{1}$.

In designs in which this element has been used, the individual steps have been made as large as $0.1 \mathrm{ohm}$, and as small as $0.000001 \mathrm{ohm}$. Elements with steps larger than $0.1 \mathrm{ohm}$ require a higher minimum resistance than is permissible in many designs, while none with steps smaller than $0.000001 \mathrm{ohm}$ have as yet been used.

Where this element is included in an instrument, the user may have no knowledge of the existence or magnitude of $r$, since all he observes directly is that when the switch is moved from any position to an adjacent one the resistance of the resistor changes by $L_{1}$. The characteristics of the element can also be described in an especially simple form by making use of the "lowering" $L$.

If $S$, without subscript, denotes the resistance of the shunt for any position of the switch, the corresponding value of $L$ is given by the expression

$$
L=\frac{r^{2}}{r+S}
$$


Differentiating (1) with $r$ constant and simplifying

$$
d L=-\frac{L^{2}}{r^{2}} d S
$$

If an electromotive force, $E$, originates in $S$, the resulting potential drop, $e$, between the terminals of the element is

$$
e=\frac{L}{r} E
$$

If the element is part of a more extended circuit, the potential drop across its terminals will be smaller than $e$ in eq 3 .

No approximations are involved in the above relations, so that they are applicable to any two resistors in parallel.

If $r$ is small compared with $S$, then approximately

and

$$
\frac{d L}{L}=\frac{2 d r}{r}(S \text { constant }),
$$

$$
\frac{d L}{L}=-\frac{d S}{S}(r \text { constant }) \text {. }
$$

Relations substantially equivalent to (1) to (5) have already been given by White.

From eq 2 it appears that a variation $d S$ in the value of $S$, which may be caused by such things as variation in the contact resistance of the switch, incorrect adjustment, or change in temperature ( $r$ remaining unchanged) will cause a change $-\left(L^{2} / r^{2}\right) d S$ in $L$. Since $L$ is largest when $S=S_{\mathrm{x}}$, the designer is most concerned with this case, and unless otherwise specified in the subsequent discussion, consideration will be limited to this case. If $L_{\mathrm{x}}$ is fixed, as it is, for example, in an element having 10 steps of $0.01 \mathrm{ohm}$ each, then $\frac{L_{\mathrm{x}}{ }^{2}}{r^{2}}$ varies inversely as $r^{2}$, so that it is doubly advantageous to make $r$ as large as permissible for a given value of $L_{x}$, up to the point where the effect of variation of contact resistance becomes negligible. In elements actually used, with one exception (example 5 of table 1) $L_{\mathrm{x}} / r$ has not exceeded $1 / 7$ so that in the most unfavorable case, $d L$ was only about $d S / 50$. Equation 2 is a special case of a general relation:

$$
d R=\left(i_{a} / i\right)^{2} d a
$$

where $R$ is the resistance of a network, $a$ is the resistance of one of the branches, and $i_{a} / i$ is the ratio of the current in the branch to the current in the network.

From eq 3 it follows that if $L / r=1 / 7$, $e$ will be equal to (or somewhat less than) $E / 7$, so that the element is inherently more effective in reducing the effect of variations of the resistance of switch contacts than it is in reducing the effect of electromotive forces originating in the switch.

From eq 4 it appears that $r$ must be adjusted with twice the proportionate accuracy required for $L_{\mathrm{x}}$, assuming that $S_{\mathrm{x}}$ is correctly adjusted, and from eq 5 that any $S$ must be adjusted to the same 
proportionate accuracy as is required for the corresponding $L$, assuming $r$ correctly adjusted. Each "A" section must be adjusted to the proportionate accuracy desired for the step to which it corresponds.

If only one element is to be included in a design, the choice of the value of $r$ is limited by two considerations. One of these is that $L_{\mathrm{x}}{ }^{2} / r^{2}$ or $L_{\mathrm{x}} / r$ shall not exceed some value determined by the requirements of the design and the quality of the switch to be used, and the other is that $r-10 L_{1}$ shall not exceed a value determined by the requirements of the design. Within the range permitted by these considerations there is usually a wide latitude for choice.

In many cases, two or more elements in series are used in a circuit, the steps of the second being 0.1 those of the first, those of the third 0.1 those of the second, etc. Such combinations will be called successive elements. Where two or more successive elements are used in one circuit, the total permissible resistance should be suitably apportioned among them. In the following discussion such considerations as simplicity in manufacture, and appropriate distribution of the permissible resistance among several successive elements will be shown to afford useful criteria for selecting values for the shunted resistances.

The values of the resistances $S_{0}, S_{1} \ldots \ldots S_{\mathrm{x}}$ and of ${ }_{1} A_{2}$, ${ }_{2} A_{3} \ldots \ldots{ }_{9} A_{\mathrm{x}}$ are defined by the following equations

$r+S_{0}=\frac{r^{2}}{0 \times L_{1}} ; r+S_{1}=\frac{r^{2}}{1 \times L_{1}} ; r+S_{2}=\frac{r^{2}}{2 \times L_{1}} \ldots r+S_{\mathrm{x}}=\frac{r^{2}}{10 \times L_{1}}$

${ }_{0} A_{1}=\frac{r^{2}}{0 \times 1 \times L_{1}} ;{ }_{1} A_{2}=\frac{r^{2}}{1 \times 2 \times L_{1}} ;{ }_{2} A_{3}=\frac{r^{2}}{2 \times 3 \times L_{1}}-\ldots{ }_{9} A_{\mathrm{x}}=\frac{r^{2}}{9 \times 10 \times L_{1}}$

It is apparent from eq 7 that if $r^{2}$ and $L_{1}$ both be multiplied or divided by the same factor, the values of the "A" sections will be unchanged. Thus one set of values for the "A" coils may be used for two or more successive elements if appropriate values are assigned to $r$ and $S_{\mathrm{x}}$ in each case.

In eq 7 all the numbers from 1 to 10 appear in the denominators. Consequently if $r^{2}$ in the numerator contains the factors 7 and 9 , the values of the "A" coils will have no recurring decimals. This suggests making $r^{2}=.63 n$, where $n$ is a number, not necessarily an integer, which is best characterized as one to be chosen with discretion. The position of the decimal point in the number so chosen for $r^{2}$ may be shifted as desired to produce appropriate values for $L_{1}$ and the "A" coils. By giving $n$ a series of values such as 1.6, 2, 2.4, $3,4,5,6,8,10,12,16,20 \ldots 160$, a series of 21 values of $r$ is obtained, each about 12 percent larger than the one preceding, the first being $1.004 \mathrm{ohms}$ and the last $10.04 \mathrm{ohms}$. From this series, a value of $r$ differing at most by 6 or 8 percent from a desired value may be selected. If, for example, an element with $0.1-\mathrm{obm}$ steps is required, in which $L_{\mathrm{x}} / r$ is not to exceed $1 / 7, n$ may be taken equal to 80 , so that $r^{2}=50.4, r=7.1, S_{\mathrm{x}}=43.3$ and the nine "A" coils beginning with ${ }_{9} A_{\mathrm{x}}$ have values of $5.6,7,9,12,16.8,25.2,42,84,252$, and $\infty$ ohms. The values given for $r$ and $S_{\mathrm{x}}$ are correct within 1 in 10000 , those for the "A" coils are exact.

A second series similar to the one above can be derived by multiplying each $n$ by 4 and shifting decimal points as required, giving the new $n$ the values $1.6,2.0,2.4,3.2,4.0,4.8,6.4,8.0,9.6,12$, 16, etc. Many 
other such series can be made, but the two given contain most of the more useful combinations.

Elements of the type described have been preferred in several instances in the design of apparatus, because of the resulting simplification in manufacture. Examples of some sets of successive elements that have been used or proposed are shown in table 1, in which values of $r^{2}$ and $n$ are tabulated. The column headed "minimum $\Sigma$ " represents the sum of the resistances of the elements when the shunts have their lowest values, corresponding in the usual case to all switches in the zero positions. The column $\Sigma L_{\mathrm{x}}^{2} / r^{2}$ shows, in the case of example 1 , that the effect of variations in the three switch contacts is $1 / 4500$ of the effect of a single switch directly in the circuit, and similarly, the column of $\Sigma L / r$ shows that the effects of electromotive forces at the three switch contacts is $1 / 50$ of the effect for a single switch directly in the circuit.

In examples 1 to 6 , the corresponding " $A$ " sections of the several elements in each example are the same. Examples 7 to 9 will be mentioned later.

TABLE 1.-Examples of successive elements

Tabulated values in columns 2 to 6 , inclusive, are those of $r^{2}$, and $n$ (in parentheses)

\begin{tabular}{|c|c|c|c|c|c|c|c|c|}
\hline \multirow{2}{*}{$\begin{array}{c}\text { Exam- } \\
\text { ple }\end{array}$} & \multicolumn{5}{|c|}{ Steps (ohms) $\left(L_{1}\right)$} & \multirow{2}{*}{$\begin{array}{l}\text { Mini- } \\
\text { mum } \Sigma \\
\text { (ohms) }\end{array}$} & \multirow{2}{*}{$\Sigma L_{\mathbf{x}}{ }^{2} / r^{2}$} & \multirow{2}{*}{$\Sigma L_{\mathrm{x}} / r$} \\
\hline & 0.1 & 0.01 & 0.001 & 0.0001 & 0.00001 & & & \\
\hline 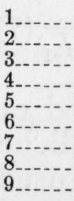 & $\begin{array}{l}0.6(20) \\
12.4(80) \\
50.6(120) \\
100 \\
35.2\end{array}$ & $\begin{array}{l}1.26(2) \\
.6048(.96) \\
1.26(2) \\
5.04(8) \\
2.52(4) \\
1 \\
8.80\end{array}$ & $\begin{array}{l}.504(.8) \\
.126(.2) \\
1.26(2) \\
.06048(.096) \\
.126(.2) \\
.504(.8) \\
.252(.4) \\
.01 \\
2.20\end{array}$ & $\begin{array}{l}.0504(.08) \\
.0126(.02) \\
.126(.2) \\
.006048(.0096) \\
.0126(.02) \\
.0504(.08) \\
.0252(.04) \\
.0001 \\
.55\end{array}$ & .00504 (.008) & $\begin{array}{l}.994 \\
1.48 \\
1.47 \\
.991 \\
4.03 \\
9.16 \\
9.83 \\
10.00 \\
10.00\end{array}$ & $\begin{array}{l}1 / 4500 \\
1 / 114 \\
1 / 11600 \\
1 / 54 \\
1 / 11.4 \\
1 / 45 \\
1 / 57 \\
1 / 25 \\
1 / 34\end{array}$ & $\begin{array}{l}1 / 50 \\
1 / 8 \\
1 / 86 \\
1 / 5.2 \\
1 / 2.5 \\
1 / 5 \\
1 / 5 \\
1 / 2.5 \\
1 / 4.8\end{array}$ \\
\hline
\end{tabular}

While sets of elements of the type illustrated in examples 1 to 6 , table 1 , have the advantage of simplifying construction and have been found satisfactory in service, it is of interest to determine whether this distribution of resistance among the elements is advantageous in other respects.

In two successive elements of this kind, the value of $L_{\mathrm{x}}{ }^{2} / r^{2}$ in the lower one is $1 / 10$ and the value of $L_{\mathrm{x}} / r$ is $1 / \sqrt{10}$ times that in the higher one. Thus in four successive elements the value of $L_{\mathrm{x}}^{2} / r^{2}$ in the lowest is 0.001 and the value of $L_{\mathrm{x}} / r$ is about 0.03 of that in the highest one. This suggests the possibility that the aggregate effects for the four elements might be appreciably reduced by a redistribution of resistance among them, subject to the condition that the sum of the shunted coils remain fixed.

It can be shown that for any number of successive elements having shunted resistances $r_{a}, r_{b}--$ etc., and subject to the condition that $\Sigma r$ is a constant, the aggregate value of $L_{\mathrm{x}}{ }^{2} / r^{2}$ will be a minimum if the values of $r$ form a geometric progression with $r_{a}=\sqrt[3]{100} r_{b}=\sqrt[3]{100^{2}} r_{c}$ etc., and the aggregate value of $L_{\mathbf{x}} / r$ will be a minimum if $r_{a}=\sqrt{10} r_{b}=$ $10 r_{c}$, etc.

Examples 6, 7, 8, and 9 of table 1 represent combinations of four elements in each of which the minimum $\Sigma$ is about 10 ohms. In 
example $8, L_{\mathrm{x}} / r$ is the same for each element. Examples 6 and 7 represent approaches to minimum values for $\Sigma L_{\mathrm{x}} / r$ and $\Sigma L_{\mathrm{x}}{ }^{2} / r^{2}$, while in example 8 the ratio of successive values of $r$ is greater, and in example 9 the ratio is less than that required for a minimum in either case. It is apparent that even the rather large departures from the condition for minimum effects involve no serious sacrifice in performance.

Furthermore, it does not follow that the combinations with the minimum values will necessarily be the optimum combinations. A selection of values involves a consideration of the purposes for which the apparatus is to be used, and in general can be made only between more or less indefinite limits. Combinations like those in the first six examples of the table 1 usually will be found to be sufficiently near optimum to make their use advantageous.

In all the elements of examples 1 to 7 of table 1 , the values of $r$ and $S_{\mathrm{x}}$ are incommensurable numbers. It is possible to make an element in which no incommensurable numbers or recurring decimals occur, by making $r=.21 n$. Such elements are not, however, as useful as the elements illustrated in examples 1 to 7 of table 1 .

The combination shown in example 1 of table 1 is substantially the same as that mentioned by Waidner and Dickinson, although the values of $r$ in their combination were slightly different, so that corresponding "A" coils were not equal. The combination of example 2 has been used in several hundred resistance-thermometer bridges.

\section{MODIFICATIONS OF THE WAIDNER-WOLFF ELEMENT}

The above discussion applies to the arrangement devised by Waidner and Wolff, and used in most of the resistance-thermometer bridges constructed in accordance with designs developed at this Bureau, in some potentiometers developed elsewhere, and in some of the apparatus formerly used in the testing of precision resistance standards. In all of the above discussion there has been an implicit assumption that the resistance introduced into a circuit by use of the elements with the dials set on lowest readings must be kept low. In this case it is advantageous to use the Waidner-Wolff arrangement in which the shunt on the highest reading is infinite. However, in many cases, the resistance is not severely limited, or the effect of contact resistance or thermal emf need not be reduced to the limit possible for a given resistance. Under these circumstances the Waidner-Wolff element may be modified by making the resistance of the shunt corresponding to the $\mathrm{X}$ or highest reading of the dial switch, of moderate instead of infinite value. This modification was first used by one of the authors (Wenner) about 1912.

In calculating resistance values for elements of this type, an extension of eq 6 and 7 may be used. Referring to figure $1 \mathrm{~B}$, the lowering need not be limited to the range from $0 L_{1}$ to $10 L_{1}$ but may be from $n L_{1}$ to $(m+n) L_{1}$ where, in practical instances, values of $n$ range from 0 to more tban 10000 . In the original Waidner-Wolff element, $n$ is 0 . Furthermore in some cases $n$ is not an integer, though $m$ is an integer equal to the number of steps of the element. Consider for example that $r$ is $60 \mathrm{ohms}$ and the resistance of the element is to be adjustable from 49.5 to $50.5 \mathrm{ohms}$ in steps of $0.1 \mathrm{ohm}$. Since the 
steps are $0.1 \mathrm{ohm}, L_{1}$ is $0.1 \mathrm{ohm}$, and the lowering, $L$, from 60 to 50.5 ohms is $95 L_{1}$ (or $n$ is 95 ). Therefore from eq 7 it follows that

$$
{ }_{95} A_{96}=\frac{3600}{95 \times 96 \times 0.1} ;{ }_{96} A_{97}=\frac{3600}{96 \times 97 \times 0.1} ;---;{ }_{104} A_{105}=\frac{3600}{104 \times 105 \times 0.1}
$$

while from eq 6 it follows that

whence

$$
S_{95}=\frac{r^{2}}{95 \times L_{1}}-r ; S_{105}=\frac{r^{2}}{105 \times L_{1}}-r
$$

The relation

$$
S_{95}-S_{105}=\frac{r^{2}}{L_{1}}\left(\frac{1}{95}-\frac{1}{105}\right)
$$

$$
S_{95}-S_{105}={ }_{95} A_{96}+{ }_{96} A_{97}+------{ }_{104} A_{105}
$$

provides a ready means of checking the arithmetical work and of determining whether the values of the " $\mathrm{A}$ " sections have been computed to a sufficient number of places.

The successive "A" sections differ from each other by only about 2 percent, and the fact that they are of moderately low and nearly equal resistance may be a material advantage in reducing the cost of construction. This and the fact that $L / r$ is nearly the same for every position of the switch are the principal advantages of the modified element over the original Waidner-Wolff element.

H. L. Curtis, of this Bureau, has designed and had constructed a number of elements which are intermediate between the element just described and the Waidner-Wolff element.

In bridges which are balanced by making small changes in one of the ratio arms, the entire resistance of the variable ratio arm may be made up of one or more elements of the kind described. In such cases it is convenient to make the resistance of each element or the sum of the resistances of all the elements an "even" value such as 50 or 100 ohms, when all dials are on their mid-setting, in order that the range of the possible change may extend an equal amount above and below the even value. The element described above has an even value of $50 \mathrm{ohms}$ for the mid-setting of the dial, which corresponds to $S_{100}$. However, it is not convenient to mark this setting 100. Furthermore it should not be marked 0 , as this would require the recording of positive and negatıve readings. A convenient marking is 5, with the markings extending from 0 to $\mathrm{X}$, and the step value marked on the instrument.

If two or more elements are connected in series, it is permissible, if the range of adjustment is small enough, to use a common $r$ for the two lower elements, as shown in figure 2. In principle the values of "A" sections of the 0.001-percent element should be different for each setting of the 0.01-percent element, and conversely. However the "A" sections of the 0.001-percent element are calculated for the mid-setting of the 0.01-percent element and vice versa. As constructed all "A" sections of the 0.001-percent element have the mean of the calculated values. Errors which may result from the effect of the setting of one element on the magnitude of the steps of the other at extreme settings amount to 0.1 step of the lower element. 
Figure 2 is a circuit diagram of a resistance ratio set, in which the ratio is adjustable from about 0.99445 to about 1.00555 (or 9.9445 to 10.0555 ) in steps of 0.001 percent. It is used in the testing of bridges and similar apparatus, and the procedure followed is such that the accuracy of the measurements depends not on ratios but on differences of ratios. Consequently the accuracy of adjustment of the principal resistance sections of the ratio set have only second-order effects on the accuracy of the measurements. Usually in a series of measurements the range in ratios is from not less than 0.998 to not more than 1.002 and then, in case the temperature is fairly constant, the quality of the apparatus is such as to justify interpolations to 0.2 step of the lowest element. Except under unusual conditions, differences of readings are reliable to one step of the lowest element, that is, differences in ratios as read from the apparatus are reliable to 1 part in 100000 of the ratio corresponding to mid-settings of all dial switches. The original apparatus bas been used in practically every test of a Wheatstone bridge, Thomson bridge, precision rheostat, and similar apparatus made in the electrical resistance laboratory during the past more than 20 years.

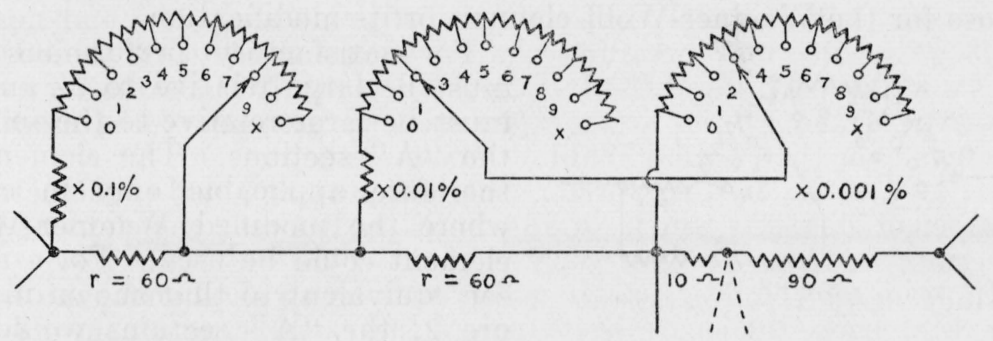

FiguRe 2.-Bridge ratio arms adjustable in steps of $0.1,0.01$, and 0.001 percent, by means of three modified Waidner-Wolff elements

Dial-switch positions are numbered from 0 to $\mathrm{X}$, though the ratio is nominally either 1 or 10 when the reading is $555 \times 0.001$-percent.

A modification of this ratio set having one more element and giving considerably higher precision was constructed in this Bureau more than 15 years ago and since then has been in regular use in the comparison of precision resistors (resistance standards). The principal resistance sections are of the double-walled sealed type containing no oil, though all resistance sections are kept in a thermostated oil bath. The switches are of good quality and operate under oil. The resistance of one section is adjustable from about 49.5555 to about $50.6665 \mathrm{ohms}$ in steps of $0.1,0.01,0.001$, and $0.0001 \mathrm{ohm}$. In use this section is connected in parallel or in series with a $50-\mathrm{ohm}$ section (or occasionally in series with a 50 - and $900-\mathrm{ohm}$ section), making an adjustable bridge arm having a nominal value of 25 or $100 \mathrm{ohms}$ (or $1000 \mathrm{ohms}$ ).

With the nominal value either 25 or $100 \mathrm{ohms}$, steps of the lowest element correspond to parts per million of the nominal value. With the nominal value $25 \mathrm{ohms}$ the current through switch contacts is approximately $1 / 22$ of the current through the bridge arm. Consequently the effect of variations of the resistance of dial switch contacts is approximately $1 / 484$ of what it would be with switch contacts directly in series. When the nominal value is $100 \mathrm{ohms}$ the effect of variations 
of the resistances of dial switch contacts, expressed in proportional parts, is the same as when it is 25 ohms.

The apparatus was intended to give directly differences of resistance ratios to a precision of 1 part per million when the ratios are within the mid-tenth of its range of adjustment which is 1.111 percent. However it has recently been used in comparing resistance standards to a precision of about 1 part in 10 million, but not without the application of calibration corrections in cases in which the range in adjustment used has been in excess of 0.05 percent.

To a limited extent both of the circuits described above are being used elsewhere.

\section{THE FIXED-SHUNT ELEMENT}

Instead of changing the resistance of the shunt, as in the elements so far described, the brush contact might be made to points between sections of the smaller resistance, leaving the shunt of fixed resistance as is shown in figure 3 . The resistances for the various sections can be calculated by direct methods, or short-cut methods can be devised if this is desired. The equations will in general be less simple than those for the Waidner-Wolff element or its modification.

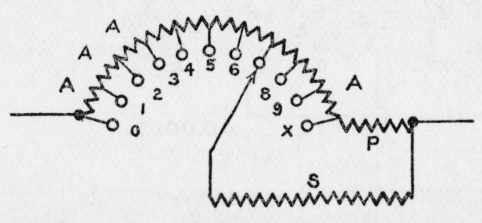

FIGURE 3.-Fixed-shunt element.

For satisfactory performance $S$ must be large relative to $P$, and $P$ must be large relative to the sum of the "A" sections. The element is therefore applicable only in cases where the modified Waidner-Wolff element could be used. For a ratio set equivalent to that shown in figure 2 , the "A" sections would be fully one order smaller and relatively more nearly equal, which may be advantageous in some cases.

A defect of this arrangement, which may or may not be serious, is that during the time the dial switch is being changed from one setting to the next higher or lower setting, it must make contact with two adjacent contact studs, thus temporarily short-circuiting one section. For the higher elements, if the current through the shunt is one-sixth the total current, the effect of short-circuiting corresponds to about three steps of the dial switch, but here it is of little consequence since in bridge (or in potentiometer) measurements, changes in settings of the dial switches of the higher elements are not made except when the battery (or galvanometer) branch is open. For the lowest element the effect would be considerably reduced by the normal resistance of connectors from junctions between "A" sections and the brush contact surfaces. For the lower elements the effect might easily be reduced to such an extent that it would be scarcely noticeable, by the addition of small resistances in the connections between "A" section junctions and switch contact surfaces, especially if a fairly quick-acting dial switch were used. While this arrangement has not been used it will probably be tried should occasion arise for constructing another adjustable ratio set for a bridge or similar apparatus. 


\section{THE MOVABLE-BRANCH-POINT ELEMENT}

A distinction, which is of some importance in the subsequent discussion, is made here between a terminal which is inherent in the element, and a lead, any number of which may be attached to any one terminal. The elements so far described may be characterized as two-terminal elements, for while the separate resistors in general have four terminals, the complete element has only two terminals. In all figures, terminals of elements or groups of elements are represented by solid circles.

The simplest of the elements having more than two terminals is the movable-branch-point element which has three terminals and is somewhat similar to the Thomson-Jenkin slide described by Varley in 1866 and to the elements used in the White two-battery potentiometer. It is shown in figure 4 in which $\mathrm{Z}$ represents a bridge arm of fairly high resistance. The "A" sections to the right (or left) of the position of the dial switch are in a bridge arm of relatively low resistance adjacent to the $\mathrm{Z}$ arm. The "A" sections to the left (or right) of the position of the switch are in the battery or galvanometer branch of the bridge. Movement of the switch transfers "A" sections to (or from) a bridge arm of fairly low resistance from (or to) the battery or galvanometer branch, while variations of the resistance of the switch contact are in the $\mathrm{Z}$ arm. For satisfactory per-

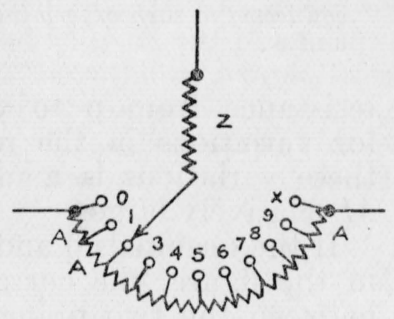

Figure 4.-Movable-branchpoint element.

formance $\mathrm{Z}$ must be sufficiently large relative to the expected variations of the resistances of the switch contacts.

In resistance-thermometer bridges constructed in accordance with designs developed in this Bureau, the $\mathrm{Z}$ arm is one of two equal ratio arms, and the current through the ratio arms is equal to that through the other arms. In typical cases the resistance of each ratio arm is about 20 times that of the adjacent low-resistance arm, consequently the effect of variations of the switch contacts or of thermoelectromotive forces in the switch is about $1 / 20$ as large as though the switch were in either of the low-resistance arms. These advantages are secured with no sacrifice of performance in other respects. The element was also used independently in a commercial type of Thomson bridge of American make, before it was used in resistance-thermometer bridges.

\section{THE PARALLEL ELEMENT}

In some resistance-measuring apparatus it is necessary to keep the sum of the resistances of the two bridge arms constant as well as to keep the effect of variations of the resistances of dial switch contacts small. In potentiometers it is necessary to keep the resistance of the battery circuit constant and in some cases to minimize the effect of variations of the resistance of switch contacts and thermoelectromotive forces in the dial-switch contacts. Consequently, if WaidnerWolff elements are used, they must be in duplicate, that is, two similar elements must be mechanically connected in such manner that as the resistance of one is increased the resistance of the other is decreased by the same amount and these two serve as a single element. 
White appears to have been the first to do this. A simpler and better arrangement is the four-terminal element shown in figure 5. All

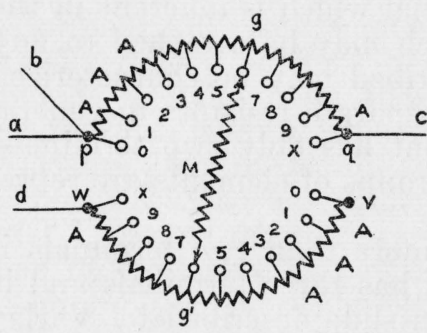

Figure 5.-Parallel element; shown with two leads connected to terminal $\mathrm{p}$, and one lead connected to each of terminals $\mathrm{q}$ and $\mathrm{w}$.

"A" sections, of which there may be any even number, are of equal resistance. The dial switch is of the double-contact type and usually the section $\mathrm{M}$ is mounted to rotate with the switch. In figure $5, \mathrm{p}, \mathrm{q}, \mathrm{w}$, and $\mathrm{v}$ represent the four terminals inherent in the element and $a, b, c$, and $d$ represent four connected leads. If a and $\mathrm{d}$ are used as current leads and $b$ and $c$ are used as potential leads, the notation $R_{\text {abcd }}$, denotes the four-terminal resistance defined by the drop in potential from $\mathrm{b}$ to $\mathrm{c}$ divided by the current entering on a and leaving on $\mathrm{d}$. This resistance is equal to $n A$ and is adjustable in steps of $A$. The resistance from $\mathrm{p}$ to $\mathrm{w}$ equals $10 A+M$, and is constant, except for variations in the resistance of switch contacts. The effect of these variations is made relatively small by making the resistance $M$ relatively large.

If the terminals $q$ and $v$ are connected by a resistance $t$, not shown in the figure, the current entering on a and leaving on $d$ divides between the two branches $\mathrm{gMg}^{\prime}$ and $\mathrm{gq} \mathrm{tvg}^{\prime}$ inversely as the resistances of these branches. If the current through $\mathrm{M}$ is the fractional part $f$, of the total current, the four-terminal resistance $R_{\text {abcd }}$ is adjustable in steps of $f A$.

\section{COMBINATIONS OF ELEMENTS}

A special feature of the parallel element is the manner in which it may be used in combination with other elements of the same or different types. Figure 6 shows one of the parallel elements (marked II) connected in series with a Hausrath six-terminal element (marked I) and in parallel with a Diesselhorst $n$-step element (marked III). Each of these three types of elements has a double-contact switch, has "A" sections of equal resistances, and the number of "A" sections is twice the number of resistance steps. Element I has six terminals, element II has four terminals, and element III has three terminals. In figure 6 , as in figure 5 , a and $\mathrm{d}$ are current leads, and $\mathrm{b}$ and $\mathrm{c}$ are potential leads. If the resistances of the " $\mathrm{A}$ " sections of element I are $1 \mathrm{ohm}$, of element II are $0.1333 \mathrm{ohm}$, and of element III are $0.04 \mathrm{ohm}$, and if the resistances $\mathrm{M}$ and $\mathrm{N}$ are so chosen that 0.75 of the current passes through $\mathrm{M}$, and 0.25 through $\mathrm{N}$, the fourterminal resistance $R_{\text {abcd }}$ will be adjustable in steps of $0.1,0.01$, and $0.001 \mathrm{ohm}$, while the resistance between $\mathrm{p}$ and $\mathrm{w}$ is constant. If the arrangement is to be used as a potentiometer, it is seen that the potential drop from any point to the left of the zero contact block of element II to any point to the right of the $\mathrm{X}$ contact block of this element, regardless of what may be to the left or the right, will be changed by this element in steps equal to the resistance of its "A" sections times the current through its dial switch. 
If $M$ is $100 \mathrm{ohms}$, then, with the most unfavorable setting of the dial switches, the resistance of the four-terminal conductor will be affected by a variation of $0.001 \mathrm{ohm}$ in the resistance of the switch contacts of element II to the extent of only 4 microhms. Furthermore, an electromotive force of 10 microvolts in the dial switch affects the potential drop from $\mathrm{b}$ to $\mathrm{c}$ by only 0.04 microvolt. It should be pointed out that the effects of variations of the resistance of dialswitch contacts, and thermoelectromotive forces in dial switches depend upon the circuit arrangement in which the parallel element (and other elements having more than two terminals) is used. The conclusions stated above concerning the effects of variations of the resistance of dial switch contacts and of thermoelectromotive forces, involve the assumption that neither has an appreciable effect on the current entering on lead a and leaving on lead d.

Figure 7 shows two of the parallel elements (elements II and III) connected in parallel with each other, in parallel with a modified Thomson-Varley slide (element I) and in parallel with a ThomsonJenkin slide (element IV). Elements I, II, and III have four ter-

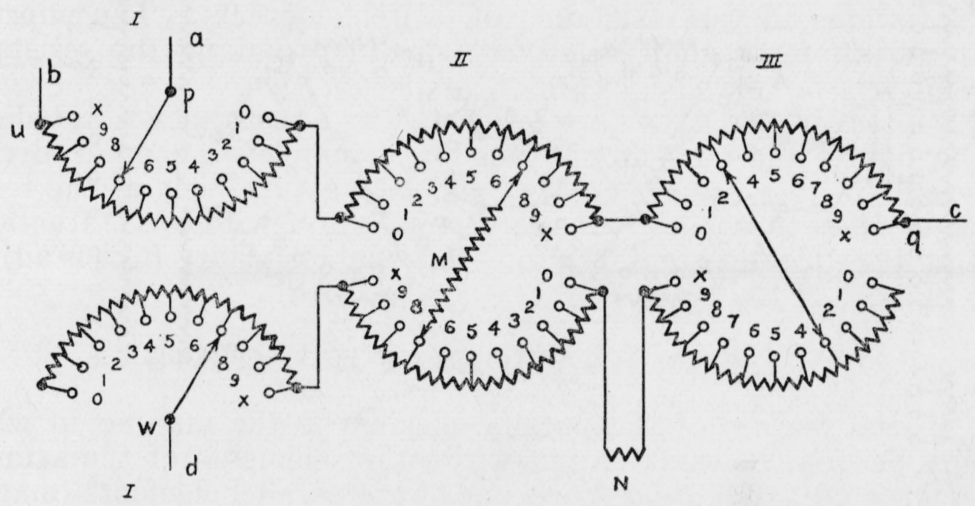

FIgURe 6.-Parallel element connected in series with a Hausrath six-terminal element and in parallel with a Diesselhorst n-step element; shown with four connected leads $\mathrm{a}, \mathrm{b}, \mathrm{c}$, and $\mathrm{d}$.

minals each, while element IV has three terminals. This arrangement constitutes two adjustable four-terminal resistances, the sum of which is constant. One of these is the ratio of the potential drop from $b$ to $h$ to the current entering on a and leaving on $d$, while the other is the ratio of the potential drop from $\mathrm{h}$ to $\mathrm{f}$ to the current entering on a and leaving on d. The former will be designated $R_{\text {abnd }}$ and the latter $R_{\text {antd. }}$. Obviously moving the double-contact switch of element I downward one step increases $R_{\text {abhd }}$ and decreases $R_{\text {ahfd }}$ by the resistance of one of the " $\mathrm{A}$ " sections of this element. By a proper choice of the resistances of " $A$ " and " $M$ " sections of elements II and III, the sections of element IV, and the " $m$ " sections between elements III and IV relative to the "A" sections of element I, a step of element II may be made 0.1 ; of element III, 0.01 ; and of element IV, 0.001 that of a step of element I. Furthermore, the effect of variations of the switch-contact resistances may be made very much less than would be the case if switches of corresponding quality were placed directly in the circuit, that is, if all instead of a relatively 
small part of the current passed through switch contacts when p and $\mathrm{w}$ are used as current terminals.

A well-known property of four-terminal resistances is that, if both current and potential leads are interchanged, the resistance is not changed. It follows therefore that the effect of variations of the resistances of the dial-switch contacts would be the same if $\mathrm{p}$ and $\mathrm{v}$ or $\mathrm{v}$ and $\mathrm{w}$, instead of $\mathrm{p}$ and $\mathrm{w}$, were used as current terminals, even though then all or a large, instead of a relatively small part of the current would pass through some of the switch contacts.

It should be noted that with the arrangement shown in figure 7 , the range over which the four-terminal resistors may be adjusted is slightly in excess of the number minus 2, of the " $\mathrm{A}$ " sections of element I, times the resistance of the "A" sections of this element. If, for example, element I has 22 sections of $10 \mathrm{ohms}$ each, the fourterminal resistances will be adjustable over a range of slightly more

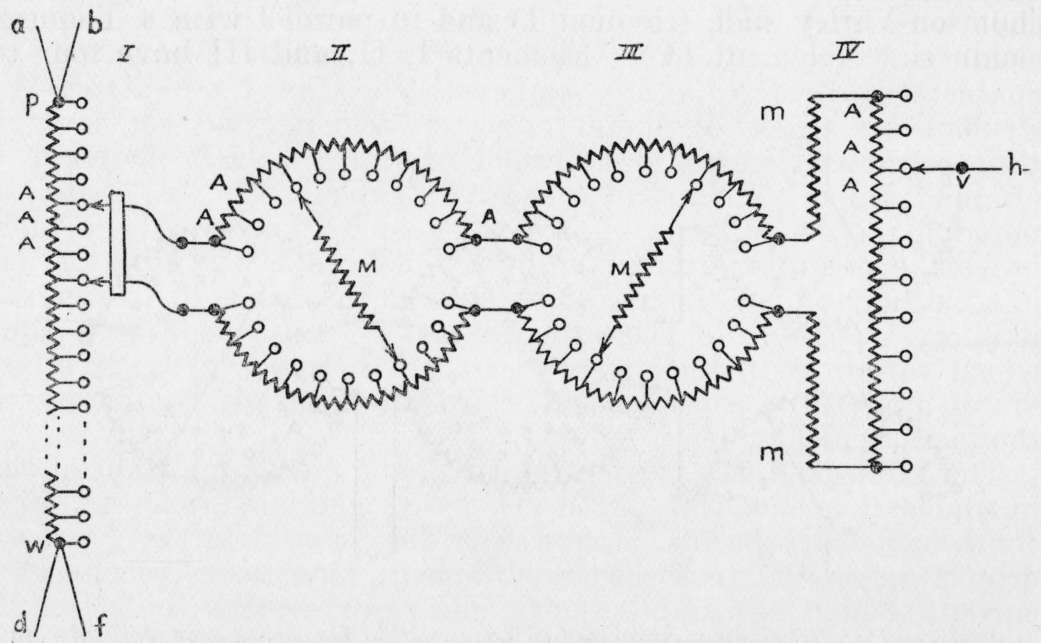

Figure 7.-Two parallel elements connected in parallel with each other, in parallel with a Thomson-Varley slide, and in parallel with a Thomson-Jenkin slide, shown with two equal resistances $\mathrm{m}$ and $\mathrm{m}$, in series with the latter, and with five connecting leads $\mathrm{a}, \mathrm{b}, \mathrm{h}, \mathrm{d}$, and $\mathrm{f}$.

than $200 \mathrm{ohms}$ in steps of $10,1,0.1$, and $0.01 \mathrm{ohms}$, while their sum will be slightly less than 220 ohms. It should be pointed out that as the dial switches of the parallel elements are changed in position, they temporarily short-circuit two "A" sections, thus temporarily lowering both of the four-terminal resistances. In cases in which this might be troublesome, the design should be such that, with $\mathrm{p}$ and w used as current terminals, the current through the " $\mathrm{M}$ " sections is large relative to that through all elements to the right (as shown in figs. 6 and 7) of the element under consideration. Failure to consider the effect of short-circuiting of "A" sections is a mistake made in the design of the first apparatus in which parallel elements were used.

Neither figure 6 nor figure 7 is intended to represent a circuit diagram for an apparatus but merely to show how a parallel element 
may be used in combination with other elements of the same or different types. While any number of parallel elements might be used, it is not expected that any case will arise in which it would be advisable to use this type of element exclusively.

\section{ELEMENTS DEVELOPED ELSEWHERE}

Consideration has been given to most of the elements devised and all the elements used in designs developed in this Bureau. Furthermore, the figures show the Hausrath six-terminal element, the Diesselhorst $n$-step element, the Thomson-Jenkin slide, and the Thomson-Varley slide. It should be pointed out that the Hausrath six-terminal element, shown in figure 6, was first described as two slide wires which served both to divide a potentiometer circuit into two parallel branches and as the lowest element of the circuit. Diesselhorst substituted a series of coils of equal resistance for each of the slide wires and, with this modification, it serves best as the highest element of a "split-circuit" potentiometer. Other elements having characteristics more or less similar to those of the Waidner-Wolff element are the Diesselhorst nine-step element and the Hausrath three-terminal element, descriptions of which may be found in the original publications and in a paper by Brooks. In this connection, mention should be made of slide wires and other types of elements in which the adjustment is continuous or substantially so rather than in a limited number of equal steps, since in the design of an apparatus it is often possible to place the contact or contacts in a branch in which variations of their resistances and thermoelectromotive forces are of little or no consequence, or are automatically compensated in the measurements.

The Lindeck-Rothe element is one of the more important of those in which the effects introduced at moving contacts are automatically compensated in the measurements. This element gives a potential drop proportional to a measured current in a fixed resistance, the current being adjustable continuously or substantially so, and measured by a milliammeter or similar deflection instrument which constitutes part of the element. By suitable design, errors which otherwise would result from thermoelectromotive forces and contact resistances may be eliminated to an extent hardly possible in most other types of elements, and for this reason the element is being used by Brooks in a number of designs intended to meet very exacting: requirements. A number of arrangements in which measurements depend upon the reading of a deflection instrument have been devised, some of which may be considered modifications of the LindeckRothe element.

Of the elements which have been used for many years, mention should be made of the element devised by $\mathrm{O}$. Wolff and said to have been used previously by Weston, which may be described as two Thomson-Jenkin slide elements mechanically connected in such a manner that as the resistance of one is increased the resistance of the other is decreased by a like amount. While in this element the effects of variations of switch contact resistances and thermoelectromotive forces are not minimized as in most other elements to which reference has been made, it has the advantage that the resistance steps may be larger than in some of the others, and this in some cases may be an 
advantage. It is used for the 10,1 , and $0.1 \mathrm{ohm}$ steps of the WolffFeussner potentiometer and could be used equally well for larger steps.

Recently both Stein, of the Leeds and Northrup Co., and Bonn, of the Rubicon Co., have proposed elements having characteristics somewhat similar to those of several of the elements considered above, but no description of either has been published.

\section{LIST OF ADJUSTABLE RESISTANCE ELEMENTS}

The adjustable resistance elements described above, and others serving more or less the same purpose, to which reference has been made, are listed below. Numbers in brackets in the list below and in the bibliography each refer to items in the other.

1. Thomson-Jenkin slide. $[13,10,6,16,17]$

2. Thomson-Varley slide. $[13,10,6,16]$

3. Waidner-Wolff element. $[14,16,7,17,12,2]$

4. Modified Waidner-Wolff element.

5. Fixed-shunt element.

6. Movable-branch-point element. [12]

7. White movable-branch-point element. $[17,2]$

8. Parallel element. $[15,5,17,1,2]$

9. Hausrath six-terminal element. $[9,10,6,7,16,17,1]$

10. Diesselhorst $n$-step element. $\quad[6,7]$

11. Hausrath three-terminal element. $[9,6,2]$

12. Diesselhorst nine-step element. $[6,10,2]$

13. Slide wires and other continuously adjustable elements.

14. Wolff element. [18]

15. Lindeck-Rothe element. $\quad[11,6,3,4,2]$

16. Stein element.

17. Bonn element.

Considering only those elements which provide both a means of adjusting a resistance and of reading the value to which it has been adjusted, this list should serve to give a good idea of the elements from which, in the design of an apparatus, a selection may be made.

\section{BIBLIOGRAPHY}

The numbers in brackets below refer to the items listed immediately above.

1. Behr, Rev. Sci. Instr. 3, 249 (1932). [8, 9]

2. Brooks, Les Potentiomètres, Cong. Int. d'Élec. Paris (1932). [3, 7, 11, 12, 15]

3. Brooks, BS J. Research 11, 211 (1933) RP586. [15]

4. Brooks and Spinks, BS J. Research 9, 781 (1932) RP506. [15]

5. Burgess and Le Chatelier, High Temperature Measurements, page 146 (1912). [8]

6. Diesselhorst, Z. Instrk. 26, 174 and 298 (1906). [1, 2, 9, 10, 11, 12, 15]

7. Diesselhorst, Z. Instrk. 28, 1 (1908). [3, 9, 10]

8. Griffiths, Nature 53, 39 (1895). [13]

9. Hausrath, Ann. Physik 322, 739 (1905). [9, 11]

10. Hausrath, Z. Instrk. 2\%, 307 (1907). [1, 2, 9, 12]

11. Lindeck and Rothe, Z. Instrk. 20, 293 (1900). [15]

12. Mueller, Bul. BS 13, 548 (1917) S288. [3,6]

13. Varley, British Assn. Rep. 36, 14 (1866). [1,2]

14. Waidner and Dickinson, Phys. Rev. 19, 57 (1904).

15. Wenner, Phys. Rev. 31, 94 (1910). [8]

16. White, Z. Instrk. $2 \%, 211$ (1907). $\quad[1,2,3,9]$

17. White, Z. Instrk. 34, 109 (1914). [1, 3, 7, 8, 9]

18. Wolff, Z. Instrk. 21, 227 (1901). [14]

Washington, August 23, 1935. 